\title{
Some Paradoxes of Mathematical Theory of Continues Mechanics
}

\author{
Evelina Prozorova \\ Mathematic and Mechanic Faculty, St. Peterburg State University, Peterhof, Russia
}

Email address:

e.prozorova@spbu.ru

To cite this article:

Evelina Prozorova. Some Paradoxes of Mathematical Theory of Continues Mechanics. American Journal of Applied Mathematics. Vol. 6, No. 1, 2018, pp. 15-19. doi: 10.11648/j.ajam.20180601.13

Received: January 11, 2018; Accepted: January 29, 2018; Published: March 7, 2018

\begin{abstract}
Abstact: The classic theory of continuum mechanics does not preserve the continuity of the environment due to the use of the conditions of equilibrium of forces and the symmetry of the stress tensor. We used many unreasonable mathematical approximations when by the Boltzmann equation is solved to describe the equations of continuum mechanics. The paper presents an analysis of mathematical approximations underlying description in different environments, and new models, to avoid the resulting misunderstandings. For rarefied gas the self-diffusion and thermo-diffusion which were foretold by S. V. Vallander are obtained from kinetic theory.
\end{abstract}

Keywords: Angular Momentum, Conservation Laws, Non-Symmetrical Stress Tensor, Boltzmann Equations, Chapman-Enskog Method, Conjugate Problem the Navie-Stokes

\section{Introduction}

The main questions of the modern mathematical theory are questions of identity to describe discrete and continuous media and to ensure continuity in the description of continua, noted A. Yu. Ishlinskii, and raised the question of the consistency of the physical parameters at the inter-element boundaries [1]. Classical mechanics of a continuous medium is based on a field description. Consequently, it deals with the distribution of points. In experiments and in computational mathematics we are dealing with elementary volume, that is, with material objects. In the first case, the rotation of the point, the distribution of the quantities inside the "point" is not important. Consequently, we discard some of the effects. Second, importantly, we actually postulate the symmetry of stresses using symmetric distribution functions, transferring the definitions of equilibrium physical quantities to the nonequilibrium case. Very important is the hypothesis of the exchange of physical quantities only along the normal, which corresponds to ignoring changes in the direction of motion within the elementary volume and the symmetry of the stress tensor. For small gradients, these approximations satisfactorily describe the processes that occur. For large gradients (turbulence, twisting of nanotubes, etc.), the adopted models do not work. A separate question is the description of discrete quantities by means of continuous quantities. The ergodic theorem does not work, whether a continuous function approximates a discrete quantity and what properties it must satisfy, it is not clear.

It is due to the use of the conditions of equilibrium of forces and the symmetry of the stress tensor. An interesting fact consists in identical the Navier-Stokes equations, obtained as limits of integrals, and through the Delta-function $[2,3]$. The present state of the classical kinetic theory can be found, for example, in $[4,5]$. Consequently really the fluxes are into the elementary volume through normal but the fluxes that through the side surfaces, we do not consider. In addition, when constructing conservation laws, we use the Stokes theorem for the passage from the integral over the surface to the integral over the volume. The theorem does not take into account the possible rotation of the elementary volume under consideration, assuming that the extraintegral term is zero. In the presence of velocity circulation, which is characteristic of a turbulent flow, the extraintegral term does not zero. In conservation laws for space coordinates averaging is fulfilled but about times is not. We have laws that are not symmetric relatively time and space. It should be noted that for the kinetic theory (the Boltzmann equation) the law of conservation of angular momentum does not hold [610]. Analysis of the recording of the Lagrangian function for 
the collective interaction of the particles with the change of the inertia center of the moving particles and the effect influence angular momentum are made in more early work.

But elementary volume can rotate around the axis of inertia or to be involved in the rotational movement. In both cases the flow density varies across the border on the value $(d(\rho u)) / d r .\left(r^{\prime}-r\right)+\cdots$ by the rotation of the elementary volume [5-9]. Macroscopic parameters are determined in the function of the Chapman-Enskog distribution which used parameters of the Euler equations. From this implies for the Chapman-Enskog equilibrium distribution function formally we have values (density, linear moment and energy) with the first-order error.

This fact was noted by Hilbert without further use and correction. The Boltzmann equation is invariant with respect to the choice of macro-parameters in equilibrium function. Therefore, the coincidence of the Navier-Stokes equations and the construction is of formal nature. Order of approximation for the parameters in a locally equilibrium distribution function is different. The new stress tensor, energy and energy flux that obtained for the molecules with their rotations and oscillations as for mixture gases are another than in classical theory. For rarefied gas the selfdiffusion and thermo-diffusion which were foretold by S. V. Vallander need to obtain from kinetic theory. So all this questions require study. In works [6-10] these questions were solved partly, but their properties were discussed not full. In work [11] the self-diffusion and thermo-diffusion were obtained from phenomenologically consideration. In this work these effects are obtained from kinetic theory. In conclusion, we note that in the construction of statistical theories of an equilibrium liquid in the Clausius theory, for pressure, eq. [12]

$$
p-n k T=-\frac{1}{6} \rho^{2} \int v(r) g(r) d r
$$

where $v(\boldsymbol{r})$ is the power of the intermolecular interaction force $\frac{d \varphi(r)}{d \ln (r)}, \varphi(r)$-potential, $g(r)=\mathrm{d} \varphi(\mathrm{r}) /(\mathrm{d} \ln (\mathrm{r}))$, and $g(\boldsymbol{r})$ is the pair distribution function; i.e. the value of the same structure (dimensional) as the term in the equations with allowance for the angular momentum, that is $M=(r-$ $\left.r_{0}\right) \times \frac{d \varphi(r)}{d r}, r_{0}-$ position of the inertia. So

$$
p-n k T=-\frac{1}{6} \rho^{2} \int\left(v(r)+r \frac{d M}{d r}\right) g(r) d r .
$$

Additional force should contribute at high temperatures. For inert gases at medium and low temperatures, the contribution is negligible.

For rarefied gas the angular momentum is value of first order. For the water molecules we have the potential for interaction of dipole and so it have some maximum and minimum, but angular momentum is main correlation effect for point molecules and another interaction is values of smaller. Then it seems probable that for pressure is to be sufficient if liquefied gases are simple, for example $\mathrm{Ar}$, of two virial coefficients and binary interaction of particles. The effect of a small distortion of circular orbits will be small due to the incommensurability the time of the rotation and displacement times of the molecules. For water, additional components will arise in connection with the asymmetry of the molecules and the perturbation of the basic potential by the interaction of the hydrogen parts with each other and with the nucleus of the second molecule.

\section{Deduction Equations with the Self-Diffusion and Thermo-Diffusion}

The main function in mechanics is the Lagrangian recorded relative to the fixed pole. However, the center of inertia moves for many material systems. Consequently, the general form of the derivative of the Lagrange function for a moving system has the form

$$
\begin{gathered}
\frac{d L}{d t}=\sum_{i}\left[\frac{\partial L}{\partial q_{i}} \dot{q}_{l}+\frac{\partial L}{\partial \dot{q}_{l}} \ddot{q}_{l}\right]+\sum_{i}\left[\frac{\partial L}{\partial\left(q_{i}-a\right)}\left(\dot{q}_{l}-\dot{a}\right)+\right. \\
\left.\frac{\partial L}{\partial\left(\dot{q}_{l}-\dot{a}\right)}\left(\ddot{q}_{l}-\ddot{a}\right)\right], \\
a=\sum_{i} \frac{m_{i} r_{i}}{m_{i}},
\end{gathered}
$$

for electrical interaction $a=\sum_{i} \frac{e_{i} r_{i}}{m_{i}}$.

Usually the Lagrange equation is written without boundary conditions.

Derivative determines the force. In addition, uneven distribution of physical quantities gives rise to the additional force due to the appearance of the angular momentum. Result:

$$
F=F_{0}+\frac{d M}{d r_{i}}
$$

where $F$ - force, acting on the particle, $F_{0}$ - the force without taking into account the angular momentum, the $M-$ the angular momentum of force acting on the particle, $r_{i}$. Equations have a higher order than the classic. Consequently, additional boundary conditions should be set. For Boltzmann equation must set boundary condition for the flow. For the equations of a continuous medium, you can specify the components of the velocity at the outer edge of the boundary layer and the velocity and vorticity. The classical kinetic theory is presented in books [13-16]. Based on the modification of the Lagrangian were derived modified Liouville equation and Boltzmann. It should be noted that the classical Boltzmann equation does not comply with the law of conservation of angular momentum. This is clearly seen if we multiply equation of speed on radius-vector of the particle to get momentum. Even with central interaction we get different values for the non-equilibrium conditions. In numerical calculations by the difference scheme using the grid pitch that is smaller the mean free path and with the ideology of a closed volume not obtain the influence of the angular momentum due to the absence of collisions. The method of molecular dynamics makes it possible to track the influence of the angular momentum if the total forces including the force from the action of the moment are 
considered. Consider the equations for the s-particle distribution function $\left(\mathrm{F}_{\mathrm{s}}\right)$ [11-14]

$$
\begin{gathered}
\frac{1}{V^{s}} \frac{\partial F_{s}}{\partial t}=-\frac{1}{V^{s}} \sum_{i=1}^{s} \frac{p_{i}}{m} \frac{\partial F_{s}}{\partial r_{i}}+\frac{1}{2 V^{s}} \sum_{\substack{i, j=1 \\
(j \neq i)}}^{s} \frac{\partial \Phi\left(\left|r_{i}-r_{j}\right|\right)}{\partial r_{i}} \frac{\partial F_{s}}{\partial \xi_{i}}+ \\
+\frac{1}{V^{s+1}} \sum_{i=1}^{s} \int \sum_{j=s+1}^{N} \frac{\partial \Phi\left(\left|r_{i}-r_{j}\right|\right)}{\partial r_{i}} \\
\frac{\partial F_{s+1}\left(t, r_{1}, \ldots, r_{s}, r_{j}, \xi_{1}, \ldots, \xi_{s}, \xi_{j}\right)}{\partial p_{i}} d r_{j} d \xi_{j}+ \\
+\frac{1}{V^{s+1}} \sum_{S+1}^{N} \int \frac{\xi_{j}}{m} \frac{\partial F_{s+1}\left(t, r_{1}, \ldots, r_{s}, r_{j}, \xi_{,}, \ldots, \xi_{s}, \xi_{j}\right)}{\partial r_{j}} d r_{j} d \xi_{j}+ \\
+\frac{1}{V^{s+1}} \sum_{i=1}^{s} \sum_{j=s+1}^{N} \int \frac{\partial \Phi\left(\left|r_{i}-r_{j}\right|\right)}{\partial r_{j}} \frac{\partial F_{s+1}\left(t, r_{1}, \ldots, r_{s}, r_{j}, \xi_{,}, \ldots, \xi_{s}, \xi_{j}\right)}{\partial p_{j}} d r_{j} d \xi_{j}+ \\
(j \neq i)
\end{gathered}
$$

It is usually assumed that when $\left|r_{j}\right| \rightarrow \infty \frac{\partial}{\partial r_{j}} F_{n} \rightarrow$ $0,\left|\xi_{j}\right| \rightarrow \infty \frac{\partial}{\partial \xi_{j}} F_{n} \rightarrow 0$. In a equilibrium stream, all physical values flows should be known.

For no equilibrium flows, such boundary conditions are not realized. The formula uses the average density, but this is suitable for relaxation problems and is not suitable for problems with large gradients. Then hypothesis about molecular chaos is break. Known solution of the ChapmanEnskog obtained using many approximations. On the other hand, the classical laws of conservation, which we consider in this part enters the normal velocity component

$$
\frac{\partial}{\partial t} \int_{\tau} \rho \delta \tau+\int_{\sigma} \rho V_{n} \delta \sigma=\int_{\tau} \dot{M} \delta \tau .
$$

For a viscous gas, the formula for the transition from the integral over the surface to the integral over the volume must be generalized, that is, the circulation of the velocity must be taken into account. Hence the Stokes formula (Ostrogradsky, Gauss) [17] should be generalized.

$$
\begin{gathered}
\iiint_{(v)}\left(\frac{\partial P}{\partial x}+\frac{\partial Q}{\partial y}+\frac{\partial R}{\partial z}\right) d v=\iint_{s}[P \cos (n, x)+ \\
Q \cos (n, y)+R \cos (n, z)] d s \\
\int_{(l)}(P d x+Q d y+R d z)=\iint_{(s)}\left[\left(\frac{\partial R}{\partial y}-\frac{\partial Q}{\partial x}\right) \cos (n, x)\right. \\
+\left(\frac{\partial P}{\partial z}-\frac{\partial R}{\partial x}\right) \cos (n, y)+ \\
\left.+\left(\frac{\partial Q}{\partial x}-\frac{\partial P}{\partial y}\right) \cos (n, z)\right] d s .
\end{gathered}
$$

For the plane case, the connection between boundary conditions is most clearly manifested after taking the integral by parts and circulation by virtue of formulas

Here $P, Q, R$ - continuous functions, $v-$ volume, $s-$ surface, $l-$ closed curve. Consequently, equation for distribution function. Consequently, in the classical theory, we ignore the circulation of velocity at the boundary of an elementary volume, which does not allow us to approach the solution of the turbulence problem. For the distribution function we have equation

$$
\begin{gathered}
f\left(t+d t, x+(\xi \cdot \mathrm{n}) \mathrm{dt}, x+(\xi \cdot \tau) d t, \xi_{i}+\frac{x_{0 i}}{m} d t\right) d x d \xi=f(t, x, \\
\left.\xi_{i}\right) d x d \xi+\left(\Delta^{+}-\Delta^{-}\right) d x \mathrm{~d} \xi \mathrm{dt}
\end{gathered}
$$

As a result, we need to get the conservation law in the form of

$$
\frac{\partial \rho}{\partial t}+\frac{\partial[(\rho u) \cdot \boldsymbol{n}+(\rho u) \cdot \boldsymbol{\tau}]}{\partial x_{i}}=0 .
$$

In classical only normal flows are considered.

Where $\mathrm{n}, \tau$ - the unit vectors along the normal and tangential to the surface; $f-$ distribution function, $x-$ coordinate, $t$ - time $\rho-$ density, $\xi$-velocity. If the Boltzmann equation is written out in the projections, more properly, all the same in the arbitrariness of the volume should be considered normal and tangential velocities. The velocity projections on the coordinate axes are used in the numerical analysis. Therefore, the error values are of the order of selfdiffusion and thermal diffusion, which will be determined by the tangential components. To understand the process of selfdiffusion and thermal-diffusion we turn to the equilibrium distribution function and investigate the effect of small additions to the values of macroscopic parameters on its value. The equilibrium function $f_{0}=$ $n^{0}\left(\frac{m}{2 \pi k T^{0}}\right)^{3 / 2} e^{-\frac{m(\xi-u)^{2}}{2 k T^{0}}}$. Let $\Delta$ is a small correction. The behavior of the function we are interested in the effect of calculating the Hilbert hypothesis macro parameters through the equilibrium distribution function.

For $\rho \cdot u$

$$
\begin{aligned}
\frac{1}{(n+\Delta n)} \int(n+ & \Delta n \\
& +\cdots)\left(\frac{m}{2(\pi k(T+\Delta T)}\right)^{\frac{3}{2}} e^{-\frac{m(\xi-u-\Delta u)^{2}}{2 k(T+\Delta T)}} d \xi .
\end{aligned}
$$

$$
\left.\int(n+\Delta n+\cdots) \xi \frac{m}{2 \pi k(T+\Delta T)}\right)^{\frac{3}{2}} e^{-\frac{m(\xi-u-\Delta u)^{2}}{2 k(T+\Delta T)}} d \xi .
$$

Formulas are expanded into series with accounting that one of formula for $\rho \cdot u$ and for $(\rho u)$ coincides. Difference among approximations is defined items at first degree of increment and have structure of the Chapman-Enskog solution.

$$
\begin{gathered}
\frac{1}{n}\left(1-\frac{\Delta n}{n}+\cdots\right) \int n\left(1+\frac{\Delta n}{n}+\cdots\right)\left(\frac{m}{2 \pi k T)}\right)^{\frac{3}{2}}(1- \\
\left.\frac{3}{2} \frac{\Delta T}{T} \ldots\right) e^{-\frac{m(\xi-u-\Delta u)^{2}}{2 k(T+\Delta T)}} d \xi= \\
\frac{1}{n}\left(1-\frac{\Delta n}{n}+\frac{\Delta n}{n}\right. \\
+\ldots)(1- \\
\left.\frac{3}{2} \frac{\Delta T}{T} \ldots\right) \int n\left(\frac{m}{2 \pi k T)}\right)^{\frac{3}{2}} e^{-\frac{m(\xi-u)^{2}}{2 k T^{0}}} e^{-\frac{m(\xi-u)^{2}}{2 k T^{0}}\left(-2(\xi-u) \Delta u-\cdots \frac{\Delta T}{T}\right)} d \xi=
\end{gathered}
$$




$$
\begin{gathered}
\frac{1}{n}(1+\ldots)\left(1-\frac{3}{2} \frac{\Delta T}{T} \ldots\right) \int n\left(\frac{m}{2 \pi k T)}\right)^{\frac{3}{2}} e^{-\frac{m(\xi-u)^{2}}{2 k T^{0}}}(1- \\
\left.\frac{m(\xi-u)^{2}}{2 k T^{0}}\left(-2(\xi-u) \Delta u-\cdots \frac{\Delta T}{T}\right)\right) d \xi \\
\frac{m(\xi-u-\Delta u)^{2}}{2 k(T+\Delta T)}=\frac{m(\xi-u)^{2}}{2 k T}(1+2(\xi-u) \Delta u+\cdots)\left(1-\frac{\Delta T}{T}\right)= \\
\left.\frac{m(\xi-u)^{2}}{2 k T}(1+2(\xi-u) \Delta u+\cdots)-\frac{\Delta T}{T}\right)
\end{gathered}
$$

For large values of the number of particles both formulas coincide. In general, we obtain values of various functions. Functionally, the Boltzmann equation is invariant with respect to the choice of macro parameters of the distribution function. It is necessary to compare the equilibrium distribution function with macroparameters taken from the Euler and Navier-Stokes equations. The difference will give us a small increment functions. We find that for the Euler equations (zero approximation of the Chapman-Enskog) the difference is zero. There are differences to the first approximation. The first approximation is responsible for the tangential component ( $p_{i j}$ tensor of viscous stresses). Euler equations are obtained with the use of locally-equilibrium distribution function. Consequently, they are responsible for the normal component of the velocity values regardless of macroparameters. Upon receipt of the first order correction of the terms included in the final decision of the ChapmanEnskog leave only after integration over the phase velocity $\xi$. The integrals are taken from $f \xi$ function, i.e. for $(\rho u)$. Consider

$$
\begin{gathered}
\frac{D f_{0}}{d t}=\frac{1}{n} f_{0} \frac{\partial n}{\partial t}+\frac{3}{2} \frac{1}{T} f_{0} \frac{\partial T}{\partial t}+\frac{m c^{2}}{2 k T^{2}} f_{0} \frac{\partial T}{\partial t}+f_{0}\left(\frac{m}{k T}(\xi-u) \frac{\partial u}{\partial t}\right)+ \\
\xi \cdot\left\{\frac{1}{n} f_{0} \frac{\partial n}{\partial x}+\left(-\frac{3}{2}\right) \frac{1}{T} f_{0} \frac{\partial T}{\partial x}+\frac{m c^{2}}{2 k T^{2}} f_{0} \frac{\partial T}{\partial x}+f_{0}\left(\frac{m}{k T}(\xi-\right.\right. \\
\left.\left.u) \frac{\partial u}{\partial x}\right)\right\}= \\
=2 J\left(f_{0}, f_{0} \varphi^{k}\right)=\int f_{0} f_{1}^{0}\left(\varphi_{1}^{(k)^{\prime}}+\varphi^{(k)^{\prime}}-\varphi_{1}^{(k)}-\right. \\
\left.\varphi^{(k)}\right) g b d b d \epsilon d \xi_{1} \xi=0 .
\end{gathered}
$$

In classic case

$$
\left.\frac{\partial f_{0}}{\partial t}\right|_{t=0}=f_{0}\left\{\frac{m}{k T}\left(c_{i} c_{j}-\frac{1}{3} c^{2} \delta_{i j}\right) \frac{\partial u_{i}}{\partial t}+\frac{1}{2 T} \frac{\partial T}{\partial t} c_{i}\left[\left(\frac{m}{k T}\right) c^{2}-5\right]\right\} .
$$

The Boltzmann equation was wrote for full function and have the local equilibrium function and addition item. The tangent velocity component is obtained because off $\xi$ have arbitrary direction of velocity relative position of coordinate axes

$$
\int n \cdot(\tau \cdot f \xi) d s d \xi=\int \operatorname{div}(\tau \cdot f \xi) d x \mathrm{~d} \xi
$$

$\tau$ give us addition item. Besides local equilibrium function $f_{0}$ we have addition item $\frac{p_{i j}}{2 p}\left(\frac{m}{2 T}\right) c_{i} c_{j}-\frac{q_{i}}{p}\left(\frac{m}{k T}\right)(1-$ $\left.\left.\frac{c^{2}}{5} \frac{m}{k T}\right) c_{i}\right]$. Main account gives derivatives of local equilibrium function. These items definite the self-diffusion and thermo-diffusion which were foretold by S. V. Vallander. The second derivative is result item $c_{i} \cdot \frac{\partial f}{\partial r_{i}}$.

\section{Definition of Macroparametrs for Multi-Components Mixture}

We can suggest the new formulation of some macrovalues (temperature, stress tensor, flow heat): in classic theory [1114] for mixture of many components gas

$$
\frac{3}{2} k T=\frac{1}{n} \sum_{k} \int \frac{m_{k} c^{k 2}}{2} f_{k} d \xi_{k},\left(\mathrm{n}=\sum_{k=1}^{N} n^{k}\right),
$$

here $k$-number of components, $T$-temperature, $c^{k}=\xi_{k}$ $\mathrm{u}$, one's velocity of molecules, $\xi_{k}$ velocity of molecules. Another definition is

$$
\frac{3}{2} k T=\frac{\int\left(\sum_{k} \frac{n_{k}}{n} m_{k}\right)\left(\sum_{\mathrm{\kappa}} \frac{n_{k}}{n} c_{k}\right)^{2}}{2} f d \xi
$$

Then we have one term is traditional and another is as the second viscosity. For stress tensor

we can have

$$
P_{i j}=\int\left(\sum_{k} \frac{n_{k}}{n} m_{k} c_{k}\right)_{i}\left(\sum_{k} \frac{n_{k}}{n} m_{k} c_{k}\right)_{j} f d \xi
$$

For the flux of the heat

$$
\mathrm{q}=\int\left(\sum_{\mathrm{K}} \frac{n_{k}}{n} c_{k}\right)_{j}\left(\sum_{\mathrm{K}} \frac{n_{k}}{n} c_{k}\right)^{2} / 2 \mathrm{f} d \xi
$$

What we take in experiment?

$$
f_{v}^{(0)}=n^{v}\left(\frac{m}{2 \pi k T}\right)^{3 / 2} \exp \left(-\frac{m}{2 k T} c^{v 2}\right)
$$

or for exclusive temperature

$$
f_{v}^{(0)}=n^{v}\left(\frac{m}{2 \pi k T^{v}}\right)^{3 / 2} \exp \left(-\frac{m}{2 k T^{v}} c^{v 2}\right) .
$$

For full temperature we cannot conclude the right probability.

\section{Conclusion}

The modified equations of continuum and Boltzmann equation in more early our works taking into account influence of angular moment and delay, as well as the position of the center of inertia of the elementary volume were made. The role of dispersion and delays in physical and chemical relaxation processes was set. The effect of angular momentum and, as a consequence, the nonsymmetrical of the stress tensor in the elementary volume was received. A model of the collective effects in the Lagrangian function was built. Now we obtain the self-diffusion and thermo-diffusion which were foretold by S. V. Vallander by phenomenological theory by kinetic theory. This is making another value for pressure of the mixture a rarefied gas and gas with internal degrees of freedom. Probably, exactly, these values are measured in all experiments. 


\section{References}

[1] A. Ye. Ishlinskii, D. D. Ivlev. The mathematical theory of plasticity.// M.: Fizmatlit, 2003, 702s.

[2] D. N. Zubarev, Nonequilibrium statistical thermodynamics.//M: Nauka, 1971. $414 \mathrm{p}$.

[3] E. P Wigner. Symmetries and reflections.//BloomingtonLondon. 1970.

[4] Fuxiang Han (2017) Kinetic Theory of Gases. Problems and Solutions in University Physics: pp. 201-223.

[5] L. Boudin, B. Grec, K. Okuda. Molecular kinetic analysis of a local equilibrium Carnot cycle. PHYSICAL REVIEW E. V. 96 N1, N. art. 012123, JUL. 2017.

[6] Evelina Prozorova. Effect of Mathematical Models on Experimental Data for the Gas and Liquids.//Journal of Mechanics Engineering and Automation. N 6, 2016, 313-318.

[7] E. Prozorova, The Role of Dispersion Effects and Delay for Continuum Mechanics.// Proceedings of 16th International Workshop on New Approaches to High-Tech: Nano-Design, Technology, Computer Simulations. NDTCS-2015, 136-8.

[8] E. V. Prozorova Influence of the Delay and Dispersion in Mechanics.// Journal of Modern Physics 2014. N5, 1796-805.

[9] E. V. Prozorova The Influence of the Dispersion of Nonequilibrium Continuum Mechanics.// Problems Environment. Moscow State University Electronic Journal: Physical and Chemical Kinetics in Gas Dynamics 13: 30. URL: http://www.chemphys.edu.ru/pdf/2012-10-30-001.pdf.

[10] Oleg Galaev, Evelina Prozorova. Dispersion Effects in the Falkner-Skan Problem and in the Kinetic Theory. Journal of Applied Mathematics and Physics, 2017, 5, 522-537.

[11] S. V. Vallander. The equations for movement viscosity gas.// DAN SSSR. 1951. V. LXX III, N 1.

[12] Physics of Simple Liquids. Edited by H. N. V Temperley, J. S. Rowlinson, G. S. Rushbrooke. Nouth-Golland publishing company. 1968. Amsterdam.

[13] C. Cercignani, Mathematical methods in kinetic theory. // Macmillan. 1969.

[14] J. H. Ferziger, H. G. Kaper, Mathematical theory of transport processes in gases.// Amsterdam-London. 1972.

[15] J. O. Hirschfelder, C. F. Curtiss, R. B. Bird, The molecular theory of gases and liquids.// New-York, 1954.

[16] M. N. Kogan. The Dynamics of the Rarefied Gases. // M.: Nauka, 1967. 440 ( Russian).

[17] Patrick Kee, David Guichard with modifications by Russ Gordon. An Introduction to Higher Mathematics 2010. 